\title{
Topological Incompleteness and Order Incompleteness of the Lambda Calculus
}

\author{
ANTONINO SALIBRA \\ Università Ca'Foscari di Venezia
}

A model of the untyped lambda calculus univocally induces a lambda theory (i.e., a congruence re-
lation on $\lambda$-terms closed under $\alpha$ - and $\beta$-conversion) through the kernel congruence relation of the
interpretation function. A semantics of lambda calculus is (equationally) incomplete if there exists
a lambda theory that is not induced by any model in the semantics. In this article, we introduce
a new technique to prove in a uniform way the incompleteness of all denotational semantics of
lambda calculus that have been proposed so far, including the strongly stable one, whose incom-
pleteness had been conjectured by Bastonero, Gouy and Berline. We apply this technique to prove
the incompleteness of any semantics of lambda calculus given in terms of partially ordered models
with a bottom element. This incompleteness removes the belief that partial orderings with a bottom
element are intrinsic to models of the lambda calculus, and that the incompleteness of a semantics
is only due to the richness of the structure of representable functions. Instead, the incompleteness
is also due to the richness of the structure of lambda theories. Further results of the article are:
(i) an incompleteness theorem for partially ordered models with finitely many connected compo-
nents (= minimal upward and downward closed sets); (ii) an incompleteness theorem for topological
models whose topology satisfies a suitable property of connectedness; (iii) a completeness theorem
for topological models whose topology is non-trivial and metrizable.

Categories and Subject Descriptors: F.3.2 [Logic and Meaninigs of Programs]: Semantics of Programming Languages-Denotational semantics; F.4.1 [Mathematical Logics and Formal Languages]: Mathematical Logic—Lambda calculus and related systems

General Terms: Languages, Theory

Additional Key Words and Phrases: Lambda calculus, Lambda theories, order and topological incompleteness, orderability/unorderability, partially ordered models, topological models

This work was partially supported by MURST Cofin'01 COMETA Project.

This article is a revised and expanded version of two papers of the author: SALIBRA, A., A continuum of theories of lambda calculus without semantics, In Proceedings of the 16th Annual IEEE Symposium on Logic in Computer Science (LICS'01), IEEE Computer Society Press, Los Alamitos, Calif., 2001a, pp. 334-343, and SAlibra, A., Towards lambda calculus order-incompleteness, In Workshop on Böhm Theorem: Applications to Computer Science Theory (BOTH 2001), Electronics Notes in Theoretical Computer Science, vol. 50, Elsevier Science Publishing Company, Amesterdam, The Netherlands, 2001b, pp. 147-160.

Author's address: Università Ca'Foscari di Venezia, Dipartimento di Informatica, Via Torino 155, 30172 Venezia, Italy; email: salibra@dsi.unive.it.

Permission to make digital or hard copies of part or all of this work for personal or classroom use is granted without fee provided that copies are not made or distributed for profit or direct commercial advantage and that copies show this notice on the first page or initial screen of a display along with the full citation. Copyrights for components of this worked owned by others than ACM must be honored. Abstracting with credit is permitted. To copy otherwise, to republish, to post on servers, to redistribute to lists, or to use any component of this work in other works requires prior specific permission and/or a fee. Permissions may be requested from Publications Dept., ACM, Inc., 1515 Broadway, New York, NY 10036 USA, fax: +1 (212) 869-0481, or permissions@acm.org.

(C) 2003 ACM 1529-3785/03/0700-0379 $\$ 5.00$ 


\section{INTRODUCTION}

Lambda theories are equational extensions of the untyped lambda calculus that are closed under derivation. They arise by syntactical considerations, a lambda theory may correspond to a possible operational (observational) semantics of the lambda calculus, as well as by semantic ones, a lambda theory may be induced by a model of lambda calculus through the kernel congruence relation of the interpretation function (see, e.g., Abramsky and Ong [1993], Barendregt [1984], and Berline [2000]). Since the lattice of the lambda theories is a very rich and complex structure, syntactical techniques are usually difficult to use in the study of lambda theories. Therefore, semantic methods have been extensively investigated.

Computational motivations and intuitions justify Scott's view of models as partially ordered sets with a least element and of computable functions as monotonic functions over these sets. After Scott, mathematical models of the lambda calculus in various categories of domains (see, e.g., Abramsky [1991]) were classified into semantics according to the nature of their representable functions (see, e.g., Barendregt [1984], Berline [2000], and Plotkin [1993]). Scott's continuous semantics [Scott 1972] is given in the category whose objects are complete partial orders and morphisms are Scott continuous functions. The stable semantics [Berry 1978] and the recent strongly stable semantics [Bucciarelli and Ehrhard 1991] are strengthenings of the continuous semantics. The stable semantics is given in the category of DIdomains with stable functions as morphisms, while the strongly stable one in the category of DI-domains with coherence, and strongly stable functions as morphisms. All these semantics are structurally and equationally rich in the sense that it is possible to build up $2^{\aleph_{0}}$ models in each of them inducing pairwise distinct lambda theories (see Kerth [1998, 2001]). The problem of the equational richness is related to the problem of the equational completeness/incompleteness of a semantics: is the set of lambda theories induced by these semantics equal or strictly included within the set of all lambda theories?

The first incompleteness result was obtained by Honsell and Ronchi della Rocca [1992] for the continuous semantics. They proved, via a hard syntactical proof, that there exists a lambda theory which cannot be the theory of a continuous model. Following a similar method, Gouy [1995] proved the incompleteness of the stable semantics with a much harder syntactical proof. Semantic and more simple proofs of incompleteness for the continuous and stable semantics can be found in Bastonero and Gouy [1999]. Bastonero [1998] provides an incompleteness result for the hypercoherence subsetting of the strongly stable semantics.

In this article, we introduce a new technique to prove in a uniform way the incompleteness of all the denotational semantics of the untyped lambda calculus which have been proposed so far. Most of the semantics which involve monotonicity with respect to some partial order fail to induce the lambda theory axiomatized by the equation $\Omega x x=\Omega$, where $\Omega$ is the usual $\lambda$-term which represents looping. The general technique used here for proving that a class $\mathcal{C}$ 
of models is incomplete is the following:

(1) Find a property $P$ satisfied by all models in $\mathcal{C}$.

(2) Find a lambda theory which is only induced by models not satisfying $P$.

We apply this technique to show the incompleteness of any lambda calculus semantics given in terms of partially ordered models with a bottom element. In particular we thus get the incompleteness of the (whole of) Bucciarelli-Ehrhard strongly stable semantics, which had been conjectured by Bastonero and Gouy [1999] and by Berline [2000], and, which was previously known only for the hypercoherence subsetting [Bastonero 1998].

The problem of the incompleteness of the semantics of lambda calculus is also related to the open problem of the order-incompleteness of lambda theories [Selinger 1996]: does there exist a lambda theory which is not induced by any nontrivially partially ordered model? Such a problem can be also characterized in terms of connected components (= minimal upward and downward closed sets) of a partial ordering: a lambda theory $\mathcal{T}$ is order-incomplete if, and only if, any partially ordered model inducing $\mathcal{T}$ has an infinite number of connected components, each one containing exactly one element of the model. Toward an answer to the order-incompleteness problem, in this article we show the existence of a lambda theory which is only induced by partially ordered models with an infinite number of connected components, each one containing at most one $\lambda$-term denotation.

Further results of the article are two topological theorems. In the first one, we show the incompleteness of any semantics given in terms of topological models whose topology satisfies a suitable property of connectedness. In the second one, a proof is given of the completeness of the semantics of lambda calculus given in terms of topological models whose topology is nontrivial and metrizable.

\subsection{Outline of the Article}

The article is organized as follows. In Section 2, we review the basic definitions of the lambda calculus and summarize definitions and results concerning topology that will be needed in the subsequent part of the article. In particular, we recall the formal definition of a model of lambda calculus, and the topological notions of connectedness and separation.

A short and simple proof of incompleteness for the class of partially ordered models with a bottom element is presented in Section 3, while the orderincompleteness problem is discussed in Section 4, where we prove the incompleteness of the class of partially ordered models with finitely many connected components.

The property of closed-open-connectedness is introduced in Section 5, where a strong property of separation orthogonal to closed-open-connectedness is proven for suitable topological algebras. This result is used to obtain the topological incompleteness theorem.

In Section 6, we show that the semantics of lambda calculus given in terms of non-trivial metric spaces is complete. 
Some open problems related to the incompleteness problem of the untyped lambda calculus are discussed in Section 7, while the last two sections of the article are devoted to related work and conclusions.

\section{PRELIMINARIES}

To keep this article self-contained, we summarize some definitions and results that we need in the subsequent part of the article. With regard to the lambda calculus we follow the notation and terminology of Barendregt [1984]. The main references for topological algebras are Taylor [1977], Bentz [1999], and Coleman [1996, 1997].

\subsection{Topology}

A topological space $(A, \tau)$ (we will occasionally avoid explicit mention of $\tau$ ) is nontrivial if there are nonempty, proper subsets $X$ and $Y$ of $A$ such that $X$ is open while $Y$ is not. If $A$ is a topological space, then the closure of a subset $U$ of $A$ will be denoted by $\bar{U}$. Recall that $a \in \bar{U}$ if $U \cap V \neq \emptyset$ for every open neighborhood $V$ of $a$. As a matter of notation, we write $\bar{b}$ for the closure of set $\{b\}$.

For any space $(A, \tau)$ a preorder can be defined by

$$
a \leq_{\tau} b \text { iff } a \in \bar{b} \text { iff } \forall U \in \tau(a \in U \Rightarrow b \in U) .
$$

In other words, $a \leq_{\tau} b$ if every neighborhood of $a$ is also a neighborhood of $b$.

We have

$$
\tau \text { is } T_{0} \text { iff } \leq_{\tau} \text { is a partial order. }
$$

For any $T_{0}$-space $A$, the partial order $\leq_{\tau}$ is called the specialization order of $\tau$. Note that any continuous map between $T_{0}$-spaces is necessarily monotone and that the order is discrete (i.e., it satisfies $a \leq_{\tau} b$ iff $a=b$ ) iff $A$ is a $T_{1}$-space.

A space $A$ is

$-T_{2}$ (or Hausdorff) if, for all distinct $a, b \in A$, there exist open sets $U$ and $V$ with $a \in U, b \in V$ and $U \cap V=\emptyset$.

$-T_{2_{1 / 2}}$ (or completely Hausdorff) if, for all distinct $a, b \in A$, there exist open sets $U$ and $V$ with $a \in U, b \in V$ and $\bar{U} \cap \bar{V}=\emptyset$.

$-T_{3}$ (or regular) if it is $T_{1}$ and, for every closed set $B \subseteq A$ and every $a \notin B$, there exist open sets $U$ and $V$ with $a \in U, B \subseteq V$ and $U \cap V=\emptyset$.

The previous axioms of separation can be relativized to pairs of elements. For example, $a$ and $b$ are $T_{2_{1 / 2}}$-separable, if there exist open sets $U$ and $V$ with $a \in U, b \in V$ and $\bar{U} \cap \bar{V}=\emptyset . T_{2^{-}}, T_{1^{-}}, T_{0^{-}}$-separability are similarly defined.

A clopen set is a subset of a space that is both open and closed. A space $A$ is connected if $A$ and $\emptyset$ are the unique clopen sets of the space $A$. The connected component of an element $a$ of a space $A$ is the greatest connected subset of $A$ including $a$. The connected components define a partition of the space $A$.

A topological algebra is a pair $(\mathbf{A}, \tau)$ where $\mathbf{A}$ is an algebra and $\tau$ is a topology on the underlying set $A$ with the property that each basic operation of $\mathbf{A}$ is continuous with respect to $\tau$. 


\subsection{Partial Orderings}

Let $(A, \leq)$ be a partially ordered set (poset). Two elements $a$ and $b$ of $A$ are: (1) comparable if either $a \leq b$ or $b \leq a$; (2) upward compatible if they have an upper bound, i.e., there exists $z$ such that $a \leq z$ and $b \leq z$; (3) downward compatible if they have a lower bound. A set $B \subseteq A$ is upward direct (downward direct) if, for all $a, b \in B, a$ and $b$ are upward (downward) compatible.

A set $B \subseteq A$ is an upward (downward) closed set if $b \in B$ and $b \leq a(a \leq b)$ imply $a \in B$. We use the notations $B \uparrow$ ( $B \downarrow$, respectively) for the least upward (downward) closed set containing a subset $B$ of $A$. We write $a \uparrow$ ( $a \downarrow$, respectively) for $\{a\} \uparrow(\{a\} \downarrow)$.

The inequality graph of a poset $(A, \leq)$ has the elements of $A$ as nodes, while an edge connects two distinct nodes $a$ and $b$ if either $a<b$ or $b<a$. Two nodes are in the same connected component if they are either not distinct or joined by a path. The equivalence classes of the relation "to be in the same connected component" define the partition of the inequality graph into connected components. A connected component can be also characterized as a minimal subset of $A$ which is both upward closed and downward closed.

Given a poset $(A, \leq)$, we can find many $T_{0}$-topologies $\tau$ on $A$ for which $\leq$ is the specialization ordering of $\tau$ (see Johnstone [1982, Sect. II.1.8]). The maximal one with this property is the Alexandroff topology, which is constituted by the collection of all upward closed sets of $A$, that is,

$$
U \text { is an Alexandroff open iff } U=U \uparrow .
$$

Then $a \uparrow$ is the least Alexandroff open set containing $a \in A$. A subset $U$ of $A$ is an Alexandroff closed set iff $U=U \downarrow$. A function is continuous with respect to the Alexandroff topology if, and only if, it is monotone. Every Alexandroff space is $T_{0}$, but not $T_{1}$, unless the order is the equality (if $x<y$, then every Alexandroff open set including $x$ includes $y$ too). Properties of the inequality graph of a poset can be expressed as topological properties of the Alexandroff topology. For example, the inequality graph of a poset is connected if, and only if, the Alexandroff topology is connected.

A partially ordered algebra, a po-algebra for short, is a pair $(\mathbf{A}, \leq)$ where $\mathbf{A}$ is an algebra and $\leq$ is a partial order on $A$ which makes the basic operations of $\mathbf{A}$ monotone. Every po-algebra is a topological algebra with respect to the Alexandroff topology.

Every $T_{0}$-topological algebra $(\mathbf{A}, \tau)$ is also a po-algebra relatively to the specialization order $\leq_{\tau}$.

\subsection{The Untyped Lambda Calculus}

The two primitive notions of the lambda calculus are application, the operation of applying a function to an argument (expressed as juxtaposition of terms), and lambda (functional) abstraction, the process of forming a function from the "rule" that defines it. The set $\Lambda$ of $\lambda$-terms of the lambda calculus over an infinite set $X$ of variables is constructed as usual: every variable $x \in X$ is a $\lambda$-term; if $M$ and $N$ are $\lambda$-terms, then so are $(M N)$ and $\lambda x . M$ for each variable $x$. 
An occurrence of a variable $x$ in a $\lambda$-term is bound if it lies within the scope of a lambda abstraction $\lambda x$; otherwise it is free. A $\lambda$-term without free variables is said to be closed. $\Lambda^{o}$ denotes the set of closed $\lambda$-terms.

$M[x:=N]$ is the result of substituting a $\lambda$-term $N$ for all free occurrences of $x$ in $M$ subject to the usual provisos about renaming bound variables in $M$ to avoid capture of free variables in $N$.

The axioms of the $\lambda \beta$-calculus are as follows: $M$ and $N$ are arbitrary $\lambda$-terms and $x, y$ are variables.

( $\alpha) \lambda x \cdot M=\lambda y \cdot M[x:=y]$, for any variable $y$ that does not occur free in $M$;

( $\beta)(\lambda x . M) N=M[x:=N]$;

The rules for deriving equations from instances of $(\alpha)$ and $(\beta)$ are the usual ones from equational calculus asserting that equality is a congruence for application and abstraction.

Lambda theories are equational extensions of the lambda calculus that are closed under derivation. If $\mathcal{T}$ is a set of equations, then the formal system $\lambda+\mathcal{T}$ is obtained by adding to $\alpha$ - and $\beta$-conversion and to the equality rules the equations in $\mathcal{T}$ as new axioms. If $\mathcal{T}$ is a set of equations, $\mathcal{T}^{+}$is the set of equations provable in $\lambda+\mathcal{T}$. $\mathcal{T}$ is a lambda theory if $\mathcal{T}^{+}=\mathcal{T}$. As a matter of notation, $\mathcal{T} \vdash M=N$ stands for $\lambda+\mathcal{T} \vdash M=N$; this is also written as $M={ }_{\mathcal{T}} N$. A lambda theory $\mathcal{T}$ is consistent if there exists at least an equation $M=N$ such that $\mathcal{T} \forall M=N$.

$\lambda \beta$ is the minimal lambda theory. $\lambda \beta \eta$ is the lambda theory generated by the axiom scheme of $\eta$-conversion:

$$
\lambda x \cdot M x=M \quad(x \text { not free in } M) .
$$

$\lambda \beta \eta$ is the minimal extensional lambda theory, since it can be also generated by adding to $\alpha$-and $\beta$-conversion and to the equality rules the following derivation rule of extensionality:

$$
M x=N x \Rightarrow M=N \quad(x \operatorname{not} \text { free in } M N) .
$$

A closed $\lambda$-term $M$ is solvable if, for every $P \in \Lambda$, there exist an integer $n$ and $N_{1}, \ldots, N_{n} \in \Lambda$ such that $M N_{1} \ldots N_{n}={ }_{\lambda \beta} P . M \in \Lambda$ is solvable if $\lambda x_{1} \ldots x_{n} . M$ is solvable, where $x_{1}, \ldots, x_{n}$ are all the variables occurring free in $M . M \in \Lambda$ is unsolvable if it is not solvable. Solvable $\lambda$-terms can be also characterized as follows: a $\lambda$-term $M$ is solvable if, and only if, it has a head normal form, that is, $M={ }_{\lambda \beta} \lambda x_{1} \cdots x_{n} . y M_{1} \cdots M_{k}$ for some $n, k \geq 0$ and $\lambda$-terms $M_{1}, \ldots, M_{k}$.

A lambda theory $\mathcal{T}$ is called semisensible [Barendregt 1984, Def. 4.1.7(iii)] if $\mathcal{T} \nvdash M=N$ whenever $M$ is solvable and $N$ is unsolvable. Every semisensible lambda theory is consistent.

Lambda theories may be defined according to syntactical considerations as well as to semantic ones. A lambda theory may be the equational theory of a model of the lambda calculus (see Section 2.5 below), or it may correspond to a possible observational semantics of the lambda calculus. In the second case, consider a subset $\mathcal{Q}$ of $\Lambda$. If $M \in \Lambda$, then we write $M \in_{\lambda \beta} \mathcal{Q}$ if there exists $P$ 
such that $M={ }_{\lambda \beta} P$ and $P \in \mathcal{Q}$. Define

$$
M \approx_{\mathcal{Q}} N \text { iff, for every context } C[], C[M] \in_{\lambda \beta} \mathcal{Q} \Leftrightarrow C[N] \in_{\lambda \beta} \mathcal{Q} .
$$

Then, the set $T h(\mathcal{Q})$, defined by

$$
\operatorname{Th}(\mathcal{Q})=\left\{M=N: M, N \in \Lambda \text { and } M \approx_{\mathcal{Q}} N\right\},
$$

is a lambda theory, called the contextual lambda theory which arises from set $\mathcal{Q}$.

\subsection{Combinatory Algebras}

Combinatory logic is a formalism for writing expressions which denote functions. Combinators are designed to perform the same tasks as $\lambda$-terms, but without using bound variables. Schönfinkel [1924] and Curry [1930] discovered that a formal system of combinators, having the same expressive power of the lambda calculus, can be based on only two primitive combinators.

We begin with the definition of a basic notion in combinatory logic and lambda calculus. An algebra $\mathbf{C}=(C, \cdot, \mathbf{k}, \mathbf{s})$, where $\cdot$ is a binary operation and $\mathbf{k}$, $\mathbf{s}$ are constants, is called a combinatory algebra (see Curry and Feys [1958]) if it satisfies the following identities (as usual the symbol · is omitted, and association is to the left):

$$
\mathbf{k} x y=x ; \quad \mathbf{s} x y z=x z(y z) .
$$

$\mathbf{k}$ and $\mathbf{s}$ are called the basic combinators. In the equational language of combinatory algebras the derived combinators $\mathbf{i}$ and $\mathbf{1}$ are defined as follows: $\mathbf{i}:=\mathbf{s k k}$ and $\mathbf{1}:=\mathbf{s}(\mathbf{k i})$. Hence, every combinatory algebra satisfies the identities $\mathbf{i} x=x$ and $1 x y=x y$.

A combinatory algebra $\mathbf{C}$ is degenerate if $C$ is a singleton set.

A function $f: C \rightarrow C$ is representable in the combinatory algebra $\mathbf{C}$ if there exists an element $c \in C$ such that $c z=f(z)$ for all $z \in C$. If this last condition is satisfied, we say that $c$ represents $f$ in $\mathbf{C}$.

Two elements $c, d \in C$ are called extensionally equal if they represent the same function in $\mathbf{C}$. For example, the elements $c$ and $\mathbf{1} c$ are extensionally equal for every $c \in C$. The combinator 1 will be used in the next section to select a canonical representative inside the class of all elements $d$ extensionally equal to a given element $c \in C$.

\subsection{Lambda Models}

Although lambda calculus has been the subject of research by logicians since the early 1930 s, its model theory developed only much later, following the pioneering model construction made by Dana Scott. The notion of an environment model (the name is due to Meyer [1982]) is described by Meyer as "the natural, most general formulation of what might be meant by mathematical models of the untyped lambda calculus". The drawback of environment models is that they are higher-order structures. However, there exists an intrinsic characterization (up to isomorphism) of environment models as an elementary class of combinatory algebras called $\lambda$-models [Barendregt 1984, Def. 5.2.7]. They 
were first axiomatized by Meyer [1982] and independently by Scott [1980]; the axiomatization, while elegant, is not equational.

We now define the notion of a $\lambda$-model. Let $\mathbf{C}$ be a combinatory algebra and let $\bar{c}$ be a new symbol for each $c \in C$. Extend the language of the lambda calculus by adjoining $\bar{c}$ as a new constant symbol for each $c \in C$. Let $\Lambda^{o}(C)$ be the set of closed $\lambda$-terms with constants from $C$. The interpretation of terms in $\Lambda^{o}(C)$ with elements of $C$ can be defined by induction as follows (for all $M, N \in \Lambda^{\circ}(C)$ and $c \in C$ ):

$$
|\bar{c}|_{\mathbf{C}}=c ;|(M N)|_{\mathbf{C}}=|M|_{\mathbf{C}}|N|_{\mathbf{C}} ;|\lambda x \cdot M|_{\mathbf{C}}=\mathbf{1} m,
$$

where $m \in C$ is any element representing the following function $f: C \rightarrow C$ :

$$
f(c)=|M[x:=\bar{c}]|_{\mathbf{C}}, \quad \text { for all } c \in C .
$$

The drawback of the previous definition is that, if $\mathbf{C}$ is an arbitrary combinatory algebra, it may happen that the function $f$ is not representable. The axioms of an elementary subclass of combinatory algebras, called $\lambda$-models or models of the lambda calculus, were expressly chosen to make coherent the previous definition of interpretation (see Meyer [1982], Scott [1980], and Barendregt [1984, Def. 5.2.7]).

The Meyer-Scott axiom is the most important axiom in the definition of a $\lambda$ model. In the first-order language of combinatory algebras, it takes the following form

$$
\forall x \forall y(\forall z(x z=y z) \Rightarrow \mathbf{1} x=\mathbf{1} y)
$$

and it makes the combinator $\mathbf{1}$ an inner choice operator. Indeed, given any $c$, the element $\mathbf{1} c$ is in the same equivalence class as $c$ with respect to extensional equality; and, by Meyer-Scott axiom, $\mathbf{1} c=\mathbf{1} d$ for every $d$ extensionally equal to $c$. Thus, the set $Y=\{c: c z=f(z)$ for all $z \in C\}$ of elements representing the function $f$ defined in (1) admits $1 \mathrm{~m}$ as a canonical representative and this does not depend on the choice of $m \in Y$.

As a matter of notation, we write $\mathbf{C} \models M=N$ for $|M|_{\mathbf{C}}=|N|_{\mathbf{C}}$.

For every $\lambda$-model $\mathbf{C}$, the lambda theory generated by the set $\{M=N$ : $M, N \in \Lambda^{o}, \mathbf{C} \models M=N$ \} is called the equational theory of $\mathbf{C}$ and is denoted by $T h(\mathbf{C})$. We say that a lambda theory $\mathcal{T}$ is induced by a $\lambda$-model $\mathbf{C}$ (or that $\mathbf{C}$ induces $\mathcal{T})$ if $\mathcal{T}=\operatorname{Th}(\mathbf{C})$.

The term model $\mathcal{M}_{\mathcal{T}}$ of a lambda theory $\mathcal{T}$ (see Barendregt [1984, Def. 5.2.11]) consists of the set of the equivalence classes of $\lambda$-terms modulo the lambda theory $\mathcal{T}$ together with the operation of application on the equivalence classes. By Corollary 5.2.13(ii) in Barendregt [1984], $\mathcal{M}_{\mathcal{T}}$ is a $\lambda$-model that induces the lambda theory $\mathcal{T}$.

A partially ordered model, a po-model for short, is a pair $(\mathbf{C}, \leq)$, where $\mathbf{C}$ is a $\lambda$-model and $\leq$ is a partial order on $C$ which makes the application operator of $\mathbf{C}$ monotone. In particular, a po-model is a po-algebra relatively to the language of combinatory algebras. A po-model $(\mathbf{C}, \leq)$ is nontrivial if the partial order $\leq$ is not discrete (i.e., $a<b$ for some $a, b \in C$ ).

A topological model of the lambda calculus is a topological algebra $(\mathbf{C}, \tau)$, where $\mathbf{C}$ is a $\lambda$-model. 


\section{PARTIALLY ORDERED MODELS WITH A BOTTOM ELEMENT}

As already mentioned, there are computational motivations for taking ordered structures with a bottom element as models of the lambda calculus. The question now arises whether every lambda theory is induced by a po-model with a bottom element. In this section, we negatively answer to this question: we exhibit a lambda theory that does not arise from such a kind of models.

Consider the lambda theory $\Pi$ axiomatized by

$$
\Omega x x=\Omega,
$$

where $\Omega \equiv(\lambda x . x x)(\lambda x . x x) . \Pi$ is semisensible ( $\Omega$ and $\Omega x x$ are both unsolvable) and then consistent.

The following lemma will be repeatedly used in the remaining part of this article.

Lemma 3.1. Let $M$ and $N$ be arbitrary $\lambda$-terms. Then we have

$$
\Pi \vdash M=N \Leftrightarrow \Pi \vdash \Omega M N=\Omega .
$$

Proof. Let $\rightarrow_{\Pi}$ be the compatible closure of the binary relation $R$ on $\Lambda$ defined as follows:

$$
R=\{(\Omega M N, \Omega): M, N \in \Lambda, \Pi \vdash M=N\} .
$$

Let $\rightarrow_{\beta \Pi}$ be the reduction rule $\rightarrow_{\beta} \cup \rightarrow_{\Pi}$. Recall that $\rightarrow_{\beta}^{*}$ is the reflexive, transitive closure of $\beta$-reduction $\rightarrow \beta$.

We divide the proof into two claims.

Claim 3.2. The relation $\rightarrow_{\beta \Pi}$ is Church-Rosser.

It is sufficient to verify that (a) the reflexive, transitive closure $\rightarrow_{\Pi}^{*}$ of $\rightarrow_{\Pi}$ satisfies the diamond property (see Barendregt [1984, Lemma 3.2.2]); (b) the relations $\rightarrow_{\beta}^{*}$ and $\rightarrow_{\Pi}^{*}$ commute (see Barendregt [1984, Def. 3.3.4]). The conclusion follows from the Hindley-Rosen Lemma (see Barendregt [1984, Prop. 3.3.5]).

Claim 3.3. $\Pi$ is the lambda theory generated by conversion $\cong_{\beta \Pi}$ from $\rightarrow_{\beta \Pi}$, that is,

$$
\Pi \vdash M=N \text { iff } M \cong_{\beta \Pi} N .
$$

If $\Omega M N \rightarrow_{\Pi} \Omega$ then we have that $\Pi \vdash M=N$ by definition of $\rightarrow_{\Pi}$, so that $\Pi \vdash \Omega M N=\Omega$ follows from $\Pi \vdash \Omega x x=\Omega$. Then it is obvious that $M \cong_{\beta \Pi} N$ implies $\Pi \vdash M=N$. For the opposite direction, it is sufficient to consider that $\Omega x x \rightarrow_{\Pi} \Omega$ for the unique axiom $\Omega x x=\Omega$ of $\Pi$.

We now conclude the proof of the lemma. If $\Pi \vdash \Omega M N=\Omega$, then $\Omega M N \cong_{\beta \Pi}$ $\Omega$, so that, by Claim 3.2, there is a reduction $\Omega M N \rightarrow_{\beta \Pi}^{*} \Omega$. This is possible only if $\Omega M N$ is a $\Pi$-redex, that is, if $\Pi \vdash M=N$.

We formally define the concepts of equational completeness/incompleteness of a class of $\lambda$-models.

Definition 3.4. A class $\mathcal{Q}$ of $\lambda$-models is called (equationally) complete if every lambda theory is induced by some $\lambda$-model of $\mathcal{Q}$. If this is not the case, $\mathcal{Q}$ is called incomplete. 
The author is indebted to Gordon Plotkin for providing the short and simple proof of Theorem 3.5 below. The original proof of the author was based on topological methods (see Salibra [2001a, 2001b]).

TheOREM 3.5. Any semantics of lambda calculus given in terms of po-models with a bottom element is incomplete.

Proof. Assume, by the way of contradiction, that the lambda theory $\Pi$ is induced by a po-model $(\mathbf{C}, \leq)$ with a bottom element $\perp$. From $\Pi \vdash \Omega x x=\Omega$, it follows that:

$$
\mathbf{C} \models \Omega x x=\Omega .
$$

Let $\mathbf{k} \equiv \lambda x y . x$ and $\mathbf{i} \equiv \lambda y . y$ (it is indeed sufficient that $\mathbf{k}$ and $\mathbf{i}$ are two distinct $\beta \eta$-normal forms). By monotonicity and by (2), the following conditions hold in $\mathbf{C}$ :

$$
\Omega \mathbf{k i} \geq \Omega \perp \perp=\Omega,
$$

and then

$$
\Omega=\Omega \Omega \Omega \leq \Omega(\Omega \mathbf{k i}) \Omega \leq \Omega(\Omega \mathbf{k i})(\Omega \mathbf{k i})=\Omega .
$$

So, we conclude:

$$
\Omega(\Omega \mathbf{k i}) \Omega=\Omega .
$$

Then, by applying twice Lemma 3.1, we get first

$$
\Omega \mathbf{k i}=\Omega,
$$

and second

$$
\mathbf{k}=\mathbf{i} .
$$

This contradicts the consistency of the lambda theory $П$.

The following corollary solves the open problem of the incompleteness of the strongly stable semantics.

Corollary 3.6. The continuous, stable, and strongly stable semantics of lambda calculus are incomplete.

We say that a semantics omits a lambda theory $\mathcal{T}$ if $\mathcal{T}$ is not induced by any model in the semantics. In Salibra [2001a], the author has shown that continuous, stable and strongly stable semantics omit a continuum of lambda theories. In fact, by applying a well-known theorem by Visser (see Visser [1980] and Barendregt [1984, Thm. 17.1.10]), it is possible to get a continuum of distinct lambda theories satisfying the conditions:

$$
\Omega x x=\Omega ; \quad \Omega(\Omega \mathbf{k i}) \Omega \neq \Omega .
$$

From the proof of Theorem 3.5, it follows that any semantics given in terms of po-models with a bottom element omits each of these lambda theories.

\section{ORDER INCOMPLETENESS}

In the previous section, we have given a negative answer to the question whether every lambda theory arises as the theory of a po-model with a bottom element. One may now relax this question by asking: does every lambda 
theory arise as the equational theory of a nontrivial po-model? The answer to this difficult question is still unknown. Selinger [1996] gave a syntactical characterization, in terms of the so-called generalized Mal'cev operators, of the order-incomplete lambda theories (i.e., the theories not induced by any nontrivial po-model). A lambda theory $\mathcal{T}$ is order-incomplete if, and only if, there exist a natural number $n \geq 1$ and a sequence $M_{1}, \ldots, M_{n}$ of closed $\lambda$-terms such that the following Mal'cev conditions are satisfied:

$$
x=\mathcal{T} M_{1} x y y ; M_{i} x x y={ }_{\mathcal{T}} M_{i+1} x y y ; M_{n} x x y=\mathcal{T} y(1 \leq i<n) .
$$

Plotkin and Simpson have shown that the above Mal'cev conditions are inconsistent with lambda calculus for $n=1$, while Plotkin and Selinger obtained the same result for $n=2$ (see Selinger [1997]). It is an open problem whether $n$ can be greater than or equal to 3 .

The problem of order-incompleteness can be also characterized as follows: a lambda theory $\mathcal{T}$ is order-incomplete if, and only if, the inequality graph (see Section 2.2) of any po-model inducing $\mathcal{T}$ has an infinite number of connected components, each one containing exactly one element of the model. In this section, we give a partial solution to the problem of order-incompleteness by showing the following result: the inequality graph of any po-model inducing the lambda theory $\Pi$ (defined in Section 3 ) has an infinite number of connected components, each one containing at most one $\lambda$-term denotation. This result implies the incompleteness of any semantics of lambda calculus given in terms of po-models with a finite number of connected components.

We begin the technical part of this section by introducing the notion of a semisubtractive algebra. These algebras satisfy a very weak form of subtractivity. We recall that the notion of subtractivity in Universal Algebra was introduced by Ursini [1994]: an algebra is subtractive, if it satisfies the identities

$$
s(x, x)=0 ; \quad s(x, 0)=x
$$

for some binary term $s$ and constant 0 . Subtractive algebras abound in classical algebras and in algebraic logic since term $s$ simulates part of subtraction. If we interpret the binary operator " $s$ " as subtraction "-" and we use the infix notation, then we can rewrite the above identities (3) as $x-x=0$ and $x-0=x$.

Definition 4.1. An algebra $\mathbf{A}$ is semisubtractive if there exist a binary term $s(x, y)$ and a constant 0 in the similarity type of $\mathbf{A}$ such that

$$
s(x, x)=0 .
$$

Example 4.2. Let $\mathbf{C}$ be a $\lambda$-model inducing the lambda theory $\Pi$ defined in Section 3. Define

$$
0 \equiv \Omega ; \quad s(x, y) \equiv \Omega x y .
$$

From $\Pi \vdash \Omega x x=\Omega$, it easily follows that $\mathbf{C} \models s(x, x)=0$. Then $\mathbf{C}$ is a semisubtractive algebra.

A semisubtractive algebra is trivial if $s(x, y)=0$ for all $x$ and $y$. Every semisubtractive po-algebra, which has both a bottom element $\perp$ and a top 
element $T$, is trivial:

$$
0=s(\perp, \perp) \leq s(x, y) \leq s(\top, \top)=0 .
$$

In the following, we always assume that a semisubtractive algebra is nontrivial.

As a matter of notation, $\omega$ denotes the first infinite ordinal, that is, the set of natural numbers.

Definition 4.3. Let $\mathbf{A}$ be a semisubtractive algebra. The subtraction sequence $\left(c_{n}\right)_{n \in \omega}$ of a pair $(a, b) \in A^{2}$ is defined by induction as follows:

$$
c_{1}=s(a, b) ; \quad c_{n+1}=s\left(c_{n}, 0\right) .
$$

We say that $(a, b)$ has order $k \in \omega$ if $c_{k} \neq 0$, while $(a, b)$ has order $\omega$ if $c_{k} \neq 0$ for all $k \in \omega$.

Notice that, if a pair $(a, b)$ has order $k$, then $a \neq b$ and $(a, b)$ has order $n$ for all $n \leq k$. In fact, if $c_{n}=0$, then $c_{n+1}=s\left(c_{n}, 0\right)=s(0,0)=0$, that is, if some $c_{n}=0$, then all $c_{j}$ with $j>n$ are 0 .

If we interpret the binary operator " $s$ " as subtraction "-" and we use the infix notation, then we can rewrite the elements of the sequence $\left(c_{n}\right)$ as follows:

$$
c_{1}=a-b ; \quad c_{2}=(a-b)-0 ; \quad c_{3}=((a-b)-0)-0 ; \quad \text { and so on. }
$$

In the ring of integers, all elements of the sequence are equal and they are all different from 0 if $a$ and $b$ are distinct.

Recall from Section 2.2 the definition of the inequality graph of a po-algebra.

LEMMA 4.4. If $(\mathbf{A}, \leq)$ is a semisubtractive po-algebra and $a$ and $b$ are joined by an edge in the inequality graph of $A$, then $s(a, b)=0$.

PROOF

$$
a \leq b \Rightarrow 0=s(a, a) \leq s(a, b) \leq s(b, b)=0,
$$

and similarly for $b \leq a$.

LEMma 4.5. Let $(\mathbf{A}, \leq)$ be a semisubtractive po-algebra. If $a, b \in$ A are joined by a path of length $k$ (in the inequality graph of $A$ ), then the pair $(a, b)$ cannot have order $k$.

Proof. The proof is by induction on the length $k$ of the path. If $k=1$, the conclusion follows from Lemma 4.4.

Assume now $a$ and $b$ to be connected by a path of length $k+1$. Then, there exists an element $d$ such that $a$ and $d$ are connected by a path of length $k$, and $d, b$ are joined by an edge (without loss of generality, we may assume $d<b$ ). Let $\left(c_{n}\right)$ be the subtraction sequence of $(a, b)$ and $\left(r_{n}\right)$ be the subtraction sequence of $(a, d)$. By the induction hypothesis, the pair $(a, d)$ does not have order $k$, so that

$$
r_{k}=0 .
$$

Since $d<b$, by an easy induction, we have that $r_{n} \leq c_{n}$ for all $n$. Then, we get

$$
0=r_{k} \leq c_{k},
$$

ACM Transactions on Computational Logic, Vol. 4, No. 3, July 2003. 
that is, 0 and $c_{k}$ are equal or joined by an edge in the inequality graph of $\mathbf{A}$. The conclusion $c_{k+1} \equiv s\left(c_{k}, 0\right)=0$ follows from Lemma 4.4.

Corollary 4.6. Let $(\mathbf{A}, \leq)$ be a semisubtractive po-algebra. If a pair $(a, b) \in$ $A^{2}$ has order $\omega$, then $a$ and $b$ are in distinct connected components.

In the next theorem, we characterize the po-models inducing the lambda theory $\Pi$ defined in Section 3. We start with a lemma.

Lemma 4.7. Let $M$ and $N$ be arbitrary $\lambda$-terms. Consider the sequence of $\lambda$-terms defined by induction as follows:

$$
c_{1} \equiv \Omega M N ; \quad c_{n+1} \equiv \Omega\left(c_{n}\right) \Omega
$$

( $c_{i}$ will become a subtraction sequence later). Then we have

$$
\Pi \nvdash M=N \Rightarrow \Pi \nvdash c_{n}=\Omega \text { for all } n .
$$

Proof. Trivial by induction on $n$, using Lemma 3.1.

Theorem 4.8. Let $(\mathbf{C}, \leq)$ be a po-model of lambda calculus. If $T h(\mathbf{C})=\Pi$, then the inequality graph of $\mathbf{C}$ has an infinite number of connected components. Moreover, if $\Pi \nvdash M=N$, then the interpretations of $M$ and $N$ in $\mathbf{C}$ are in distinct connected components.

Proof. By Example 4.2, $\mathbf{C}$ is a semisubtractive algebra, so that by Lemma 4.7 any pair $(M, N)$ of two non- $\Pi$-equivalent closed $\lambda$-terms has or$\operatorname{der} \omega$. Then, by Corollary 4.6 $M$ and $N$ are in distinct connected components of the inequality graph of $\mathbf{C}$. Since $\Pi$ is consistent there are infinitely many pairwise non- $\Pi$-equivalent closed $\lambda$-terms (for example, the non- $\beta \eta$-equivalent normal forms), and then infinite distinct connected components.

Theorem 4.9 (Order Incompleteness Theorem). Any semantics of the untyped lambda calculus, given in terms of po-models that have only finitely many connected components, is incomplete.

Proof. By Theorem 4.8.

Corollary 4.10. Any semantics of lambda calculus given in terms of pomodels which are semilattices, lattices, complete partial orderings, or which have a top or a bottom element, is incomplete.

\section{TOPOLOGICAL INCOMPLETENESS}

In this section, we show the incompleteness of any semantics of lambda calculus, given in terms of topological models satisfying a suitable property of connectedness called closed-open-connectedness. The proof is based on a general theorem of separation for topological algebras.

\subsection{A Topological Theorem of Separation}

We recall that separation axioms in topology stipulate the degree to which distinct points may be separated by open sets or by closed neighbourhoods of open sets. In Theorem 5.2 below, we prove that in every semisubtractive 
$T_{0}$-topological algebra every pair of elements of order 3 is $T_{2_{1 / 2}}$-separable. We were inspired with Bentz and Coleman for the idea of this theorem and for the techniques used in its proof (see Bentz [1999] and Coleman [1996, 1997]).

THEOREM 5.1. If $(\mathbf{A}, \tau)$ is a semisubtractive $T_{0}$-topological algebra, then every pair $(a, b) \in A^{2}$ of order 2 is $T_{2}$-separable.

Proof. Let $\left(c_{n}\right)$ be the subtraction sequence of $(a, b)$. Since $\mathbf{A}$ is a po-algebra with respect to the specialization ordering $\leq_{\tau}$ of $\tau$ (see Section 2.2), then, from the hypothesis $c_{2}=s\left(c_{1}, 0\right) \neq 0$ and from Lemma 4.4, it follows that $c_{1}$ and 0 are incomparable with respect to $\leq_{\tau}$, that is, neither $c_{1} \leq_{\tau} 0$ nor $0 \leq_{\tau} c_{1}$. Then, there exists an open neighborhood $U$ of $c_{1}$ such that $0 \notin U$.

From $s(a, b)=c_{1} \in U$ and from the continuity of $s$, it follows that there exist two open neighborhoods $V, W$, of $a$ and $b$ respectively, such that $s(V, W) \subseteq U$. If there is an element $e \in V \cap W$, then $0=s(e, e) \in U$. This contradicts the hypothesis on $U$. Hence, we have $V \cap W=\emptyset$.

Theorem 5.2. If $(\mathbf{A}, \tau)$ is a semisubtractive $T_{0}$-topological algebra, then every pair $(a, b) \in A^{2}$ of order 3 is $T_{2_{1 / 2}}$-separable.

Proof. Let $\left(c_{n}\right)$ be the subtraction sequence of $(a, b)$ and $\left(d_{n}\right)$ be the subtraction sequence of $\left(c_{1}, 0\right)$. It is obvious that we have $d_{n}=c_{n+1}$ for all $n$ and, hence, that $\left(c_{1}, 0\right)$ has order 2 . By applying Theorem 5.1 , we get an open neighborhood $V^{\prime}$ of $c_{1}$ and an open neighborhood $W^{\prime}$ of 0 such that $V^{\prime} \cap W^{\prime}=\emptyset$.

Since $s$ is continuous and $s(a, b)=c_{1} \in V^{\prime}$, there exist two other open sets $V$ and $W$ containing $a$ and $b$, respectively, such that $s(V, W) \subseteq V^{\prime}$. The sets $V$ and $W$ will be the right sets for the conclusion of the theorem. Since $s$ is continuous the pre-image of $\overline{V^{\prime}}$ under the map $s$ is closed. From $s(V, W) \subseteq V^{\prime} \subseteq \overline{V^{\prime}}$, the pre-image of $\overline{V^{\prime}}$, which is closed, contains $V \times W$, so $s(\bar{V}, \bar{W}) \subseteq \overline{V^{\prime}}$.

We now prove that $\bar{V} \cap \bar{W}=\emptyset$. Assume, by the way of contradiction, that there is $e \in \bar{V} \cap \bar{W}$. Since $s(\bar{V}, \bar{W}) \subseteq \overline{V^{\prime}}$, it follows that $0=s(e, e) \in \overline{V^{\prime}}$. But by definition of closure of a set this is possible only if, for every open neighbourhood $Z$ of 0 , we have that $Z \cap V^{\prime} \neq \emptyset$. But this contradicts our initial choice of $V^{\prime}$ and $W^{\prime}$ as two open neighborhoods of $c_{1}$ and 0 respectively with empty intersection.

\subsection{Closed-Open-Connectedness}

Connectedness axioms in topology examine the structure of a topological space in an orthogonal way with respect to separation axioms. They deny the existence of certain subsets of a topological space with properties of separation. In this section, we introduce a strong property of connectedness, called closed-openconnectedness, which is orthogonal to the property of $T_{2_{1 / 2}}$-separability, and it is satisfied by a topological space if there exist no $T_{2_{1 / 2}}$-separable elements.

Definition 5.3. We say that a space is closed-open-connected, co-connected for short, if it has no disjoint closures of open sets. In other words, if, for all open sets $U$ and $V$, we have that $\bar{V} \cap \bar{U} \neq \emptyset$.

We recall that a space with no disjoint open sets is called hyperconnected, while a space with no disjoint closed sets is called ultraconnected (see Steen 
and Seebach [1978, Sect. 4]). Then, we have the following implications:

$$
\text { hyperconnectedness } \Rightarrow \text { co-connectedness } \Rightarrow \text { connectedness }
$$

and

$$
\text { ultraconnectedness } \Rightarrow \text { co-connectedness } \Rightarrow \text { connectedness. }
$$

Then closed-open-connectedness is a sort of meeting point between ultraconnectedness and hyperconnectedness.

The following proposition provides a wide class of topological spaces whose topology is co-connected.

Proposition 5.4. Let $(X, \tau)$ be a $T_{0}$-topological space, whose specialization order $\leq_{\tau}$ satisfies the following property: every pair of nodes of the inequality graph of $\left(X, \leq_{\tau}\right)$ is joined by a path of length less or equal to 3 . Then $(X, \tau)$ is co-connected.

Proof. Assume, by the way of contradiction, that there exist elements $a, b \in$ $X$ and open sets $U$ and $V$ such that $a \in U, b \in V$ and $\bar{V} \cap \bar{U}=\emptyset$. Then $a$ and $b$ are incomparable with respect to the specialization ordering; otherwise, either $a \in V$ or $b \in U$. By hypothesis, $a$ and $b$ are joined by a path of length less or equal to 3 . We have four possibilities.

(1) ( $\exists c$ ) $a<c>b$. In this case, $c \in U \cap V$.

(2) ( $\exists c) a>c<b$. Then, we have $c \in \bar{a} \subseteq \bar{U}$ and $c \in \bar{b} \subseteq \bar{V}$.

(3) $(\exists c, d) a<c>d<b$. Then, $d \in \bar{c} \subseteq \bar{U}$ and $d \in \bar{b} \subseteq \bar{V}$.

(4) $(\exists c, d) a>c<d>b$. Then, $c \in \bar{d} \subseteq \bar{V}$ and $c \in \bar{a} \subseteq \bar{U}$.

All these possibilities contradict our assumption.

Corollary 5.5. Every $T_{0}$-topological space $(X, \tau)$, whose specialization order either admits a bottom (top) element or makes $X$ an upward (downward) direct set, is co-connected.

In particular, complete partial orderings (including continuous, stable and strongly stable models) with the Scott topology are co-connected.

We cannot improve Proposition 5.4. The following counter-example provides a space, whose topology is not co-connected, and such that every pair of nodes of the inequality graph associated with the specialization order $\leq_{\tau}$ is joined by a path of length less or equal to 4 . Let $X=\{a, b, c, d, e\}$ be a set partially ordered as follows: $a>b<c>d<e$. Then $X$ is a topological space with respect to the Alexandroff topology (see Section 2.2). $X$ is not co-connected, since $a \uparrow \downarrow=\{a, b\}$ and $e \uparrow \downarrow=\{d, e\}$ are disjoint closures of the open sets $a \uparrow$ and $e \uparrow$.

\subsection{The Topological Incompleteness Theorem}

As a consequence of the theorem of separation of Section 5.1, we show that the topological models of the lambda theory $\Pi$ are not co-connected. The topological incompleteness theorem follows from this result.

Recall from Section 2.5 that a topological model of the lambda calculus is a topological algebra $(\mathbf{C}, \tau)$, where $\mathbf{C}$ is a $\lambda$-model. 
Theorem 5.6. Let $(\mathbf{C}, \tau)$ be a $T_{0}$-topological model of the lambda calculus. If $T h(\mathbf{C})=\Pi$ then $\tau$ is not co-connected .

Proof. We recall from Example 4.2 that $\mathbf{C}$ is a semisubtractive algebra. Let $\mathbf{k} \equiv \lambda x y . x$ and $\mathbf{i} \equiv \lambda y . y$ (it is indeed sufficient that $\mathbf{k}$ and $\mathbf{i}$ are two distinct $\beta \eta$-normal forms). Then the subtraction sequence of $(\mathbf{k}, \mathbf{i})$ has order $\omega$ by Lemma 4.7, so that $\mathbf{k}$ and $\mathbf{i}$ are $T_{2_{1 / 2}}$-separable by Theorem 5.2.

We conclude this section with the topological incompleteness theorem.

Theorem 5.7 (Topological Incompleteness Theorem). Any semantics of the lambda calculus given in terms of co-connected $T_{0}$-topological models is incomplete.

Proof. From Theorem 5.6.

We now analyse the relationships between the order incompleteness theorem and the topological incompleteness theorem.

Recall from Section 2.1 that a topological model, whose topology is $T_{0}$ but not $T_{1}$, has a non-trivial specialization order $(a<b$ for some $a, b)$. Then, from Theorem 4.9 and from Theorem 5.7, it follows the incompleteness of any semantics of lambda calculus given in terms of $T_{0}$-topological models, whose topology is either co-connected or admits a specialization order with a finite number of connected components.

Partial orderings can be viewed as topological spaces with respect to the Alexandroff topology (see Section 2.2). Then the order incompleteness theorem can be also expressed as follows (see Salibra [2001b]): any semantics of lambda calculus given in terms of Alexandroff topological models with a finite number of connected components is incomplete.

All semantics we have shown incomplete in Corollary 4.10 are constituted by po-models whose Alexandroff topology is co-connected (see Corollary 5.5). Then, the incompleteness of continuous, stable and strongly stable semantics also follows from the topological incompleteness theorem.

The following natural questions arise:

(Q1) Is there a po-model of lambda calculus whose Alexandroff topology is connected but not co-connected?

(Q2) Is there a po-model of lambda calculus whose Alexandroff topology has a finite number (greater than 1) of connected components?

(Q3) Is there a nontrivial co-connected $T_{1}$-topological model of lambda calculus? In other words, is there a co-connected topological model of lambda calculus, whose specialization order is the equality relation?

\section{COMPLETENESS}

In this section, we positively answer the following question: is there a class of $\lambda$-models with a "reasonable" topology which is a complete semantics of lambda calculus? In the main result of this section, we show that the semantics of lambda calculus, given in terms of topological models whose topology is nontrivial and metrizable, is complete. We conclude the section by discussing some 
negative results about the existence of topological models in categories of topological spaces.

If $(A, d)$ is a metric space, then $\tau_{d}$ is the topology on $A$ defined by $d$. A metrizable model of the untyped lambda calculus is a topological model whose topology is non-trivial and metrizable.

Theorem 6.1 (Metric Completeness Theorem). The semantics of the untyped lambda calculus given in terms of metrizable models is complete.

Proof. Let $\mathcal{T}$ be an arbitrary consistent lambda theory. The term model $\mathcal{M}_{\mathcal{T}}$ of $\mathcal{T}$ defined in Section 2.5 is a $\lambda$-model [Barendregt 1984, Corollary 5.2.13(ii)] such that $T h\left(\mathcal{M}_{\mathcal{T}}\right)=\mathcal{T}$. We show that it is possible to define on $\mathcal{M}_{\mathcal{T}}$ a metric $d$ in such a way that $\left(\mathcal{M}_{\mathcal{T}}, \tau_{d}\right)$ is a metrizable model. Consider the closed term model $\mathcal{M}_{\mathcal{T}}^{o}$ of $\mathcal{T}$, that is the subalgebra of $\mathcal{M}_{\mathcal{T}}$ generated by the equivalence classes of closed $\lambda$-terms. Notice that $\mathcal{M}_{\mathcal{T}}^{o}$ is not in general a $\lambda$-model (see Barendregt [1984, Corollary 5.2.13(i)]).

Let $X$ be the set of variables of lambda calculus. Then $\mathcal{M}_{\mathcal{T}}$ is the free extension of $\mathcal{M}_{\mathcal{T}}^{o}$ by $X$ in the variety of combinatory algebras (see, e.g., Pigozzi and Salibra [1998, Sect. 3.3] and Salibra [2000, Sect. 4.2]), that is, $\mathcal{M}_{\mathcal{T}}$ satisfies the following universal mapping property: for every homomorphism $h: \mathcal{M}_{\mathcal{T}}^{o} \rightarrow \mathbf{A}$ from $\mathcal{M}_{\mathcal{T}}^{o}$ into a combinatory algebra $\mathbf{A}$ and every mapping $g: X \rightarrow A$ there exists a unique homomorphism $f: \mathcal{M}_{\mathcal{T}} \rightarrow \mathbf{A}$ of combinatory algebras extending both $h$ and $g$.

Since $\mathcal{M}_{\mathcal{T}}$ is a free algebra, then we can apply the following construction due to Świerczkowski [1964] (see also Taylor [1977, Thm. 2.1] and Coleman [1997, Sect. 3]). Let $\left(X, d_{0}\right)$ be an arbitrary metric space whose universe $X$ is the set of variables of lambda calculus. By applying Świerczkowski's theorem, it is possible to extend the metric $d_{0}$ to a metric $d$ on the free algebra $\mathcal{M}_{\mathcal{T}}$ in such a way that the application operator is continuous with respect to $\tau_{d}$ and $d(x, y)=d_{0}(x, y)$ for all $x, y \in X$. The conclusion of the theorem follows because $\left(\mathcal{M}_{\mathcal{T}}, \tau_{d}\right)$ is a metrizable model of $\mathcal{T}$.

Are there other classes of topological models that are complete semantics of the lambda calculus? In order to answer this question, it is necessary to consider the properties of separation of the topological models of the lambda theory $\Pi$ (see Theorem 5.6). We recall that many authors tried to find lambda calculus models in Cartesian closed categories of topological spaces. Abramsky (see Mislove [1998, Thm. 5.11]) and Plotkin (see Mislove [1998, Thm. 5.14]) have shown respectively that there exists no nondegenerate model of the lambda calculus in the category of posets and monotone mappings, and in the category of complete ultrametric spaces and nonexpansive mappings. Hofmann and Mislove [1995] have shown that the category of $k$-spaces and continuous maps has no nondegenerate, compact $T_{2}$-topological model. A $k$-space is a topological space in which a subset is open if and only if its intersection with each compact subset of the space is open in the subspace. The following problem is still open.

(Q4) (Hoffman-Mislove) Is there a lambda calculus model in the category of $k$-spaces? 
Notice that every topological $\lambda$-model, in which all the continuous selfmaps of the model are representable (as in the category of $k$-spaces), must have a connected topology because of the existence of fixed points. If a topology is not connected, then there exists a clopen (open and closed) set $U$ and two elements $a$ and $b$ such that $a \in U$ and $b \notin U$. Then, the function $f$, defined by

$$
f(x)=b \text { if } x \in U ; \quad f(x)=a \text { if } x \notin U,
$$

is continuous without fixed points. The following natural question now arises:

(Q5) Is the semantics of lambda calculus, given in terms of connected topological models, complete?

\section{ORDERABILITY/UNORDERABILITY}

In this section, we present some further results and discuss some open problems related to the incompleteness problem of the untyped lambda calculus.

If every model of lambda calculus were nontrivially orderable, then the orderincompleteness problem (discussed in Section 4) would admit a negative answer. However, Selinger [1996] has recently shown that the term models $\mathcal{M}_{\lambda \beta}$ and $\mathcal{M}_{\lambda \beta \eta}$ of the lambda theories $\lambda \beta$ and $\lambda \beta \eta$ are unorderable (i.e., they do not admit a nontrivial compatible partial order). As a consequence of the theorem of separation in Section 5.1, we now show that the term model $\mathcal{M}_{\Pi}$ of the lambda theory $\Pi$ is also unorderable.

Definition 7.1. An algebra $\mathbf{A}$ is strongly semisubtractive if it is semisubtractive and satisfies the following condition:

$$
s(x, y)=0 \Rightarrow x=y .
$$

Proposition 7.2. A strongly semisubtractive $T_{0}$-topological algebra $\mathbf{A}$ is unorderable.

Proof. Assume, by the way of contradiction, that there exists a nontrivial compatible partial order on A. Let $a, b \in A$ be distinct elements with $a<b$. Then, from Lemma 4.4, it follows that $s(a, b)=0$, which implies $a=b$ by (5). Contradiction.

COROLLARY 7.3. $\mathcal{M}_{\Pi}$ is strongly semisubtractive, and hence unorderable.

Proof. By Lemma 4.7 and by Proposition 7.2.

Proposition 7.4. A strongly semisubtractive $T_{0}$-topological algebra $\mathbf{A}$ is $T_{2_{1 / 2}}$.

Proof. From (5), it follows that every pair of distinct elements of $A$ has order $\omega$. Then, the conclusion follows from Theorem 5.2.

It is not possible to improve the conclusion of Proposition 7.4, as shown by the following example suggested to the author by E. Jabara.

Example 7.5. Let $R$ be the set of real numbers, $A=\{(x, y) \mid x, y \in R, y \geq$ $0\}$ be the upper halfplane of $R^{2}$ and $\tau_{0}$ be the usual Euclidean topology on $A$. 
For every $(a, 0) \in A$ and for every real number $\epsilon>0$, define

$$
K_{a}(\epsilon)=\left\{(x, y) \in A \mid(x-a)^{2}+y^{2}<\epsilon, y>0\right\} \cup\{(a, 0)\} .
$$

If $\tau$ is the least topology on $A$ containing all the elements of $\tau_{0}$ and all $K_{a}(\epsilon)$ (for every $a, \epsilon \in R$ with $\epsilon>0$ ), then it is possible to verify that $(A, \tau)$ is a $T_{2_{1 / 2}}$-space but not a $T_{3}$-space. The binary operation $\star$ on $A$, defined by

$$
\left(x_{1}, y_{1}\right) \star\left(x_{2}, y_{2}\right)=\left(x_{1}-x_{2},\left|y_{1}-y_{2}\right|+1\right),
$$

is continuous with respect to the topology $\tau$, and it simulates part of the subtraction (with " 0 " represented by the pair $(0,1)$ ):

$$
\left(x_{1}, y_{1}\right) \star\left(x_{2}, y_{2}\right)=(0,1) \text { iff } x_{1}=x_{2}, y_{1}=y_{2} .
$$

Then $(A, \star, \tau)$ is a strongly semisubtractive topological algebra whose topology is not $T_{3}$.

The classification of the $\lambda$-models into orderable/unorderable models can be refined as follows. For every model $\mathbf{C}$, let $T_{i}^{\mathbf{C}}\left(i=0,1,2,2_{1 / 2}\right)$ be the set of all topologies $\tau$ on $C$ which make $(\mathbf{C}, \tau)$ a $T_{i}$-topological model. It is obvious that, in general, we have

$$
T_{0}^{\mathbf{C}} \supseteq T_{1}^{\mathbf{C}} \supseteq T_{2}^{\mathbf{C}} \supseteq T_{2_{1 / 2}}^{\mathbf{C}} .
$$

We recall from Section 2.1 that a topology with a nontrivial specialization order (we have $a<b$ for some $a, b$ ) would be $T_{0}$ yet not $T_{1}$, so that

$$
\mathbf{C} \text { is unorderable iff } T_{0}^{\mathbf{C}}=T_{1}^{\mathbf{C}} \text {. }
$$

We say that a lambda theory $\mathcal{T}$ is of (topological) type $i\left(i=0,1,2,2_{1 / 2}\right.$ ) if the term model of $\mathcal{T}$ satisfies $T_{0}^{\mathcal{M}_{\mathcal{T}}}=T_{i}^{\mathcal{M}_{\mathcal{T}}}$. All lambda theories are of type 0 ; the lambda theory $\mathcal{B}$, generated by equating two lambda terms if they have the same Böhm tree, is not of type 1 (see Barendregt [1984]). $\lambda \beta$ and $\lambda \beta \eta$ are of type 1 by Selinger's result, while $\Pi$ is of type $2_{1 / 2}$ by Corollary 7.3 and by Proposition 7.4. Then, some natural questions arise:

(Q6) Is $\lambda \beta(\lambda \beta \eta)$ of type 2 ?

(Q7) Is $\lambda \beta(\lambda \beta \eta)$ of type $22_{1 / 2}$ ?

Selinger [1996, 1997] has shown that the problem of the orderincompleteness is also related to the following question by Plotkin [1996]:

(Q8) Is there an absolutely unorderable combinatory algebra?

A combinatory algebra is absolutely unorderable if it cannot be embedded in any nontrivially partially ordered combinatory algebra. In the last result of this Section we give a partial positive answer to Plotkin's question (see Lusin and Salibra [2003] for a different approach to this problem).

We recall that a quasi-variety is a class of algebras axiomatized by quasiidentities (i.e., equational implications with a finite number of equational premises). 
Definition 7.6. Let $\mathcal{V}$ be a quasi-variety of algebras and let $\mathbf{C} \in \mathcal{V}$. We say that $\mathbf{C}$ is absolutely unorderable in $\mathcal{V}$ if, for every embedding $\mathbf{C} \hookrightarrow \mathbf{D} \in \mathcal{V}, \mathbf{D}$ is unorderable.

Proposition 7.7. Let $\mathcal{S D}$ be the quasi-variety of combinatory algebras axiomatized by $\Omega x y=\Omega \Leftrightarrow x=y$. Then, the term model $\mathcal{M}_{\Pi}$ of the lambda theory $\Pi$ is absolutely unorderable in $\mathcal{S D}$.

Proof. $\mathcal{M}_{\Pi}$ belongs to $\mathcal{S D}$ because of Corollary 7.3. Then, the conclusion follows from Proposition 7.2 since all algebras in $\mathcal{S D}$ are strongly semisubtractive.

\section{RELATED WORK}

In this section, we review the classic results of incompleteness for the continuous and stable semantics. The first incompleteness result was obtained by Honsell and Ronchi della Rocca [1992] for the continuous semantics. They extended to all continuous models arising from an inverse limit construction an approximation theorem introduced by Hyland and Wadsworth to study the Scott model $D_{\infty}$ (see Barendregt [1984]). The general approximation theorem was applied to prove that the lambda theory induced by the Park continuous model $\mathbf{P}$ is included within the contextual lambda theory $T h\left(\Lambda^{o}\right)$ induced by the set $\Lambda^{\circ}$ of closed $\lambda$-terms (see Section 2.3 for the definition of contextual lambda theory). Then, the authors prove the incompleteness of the continuous semantics by showing that, whenever the theory of a continuous model is included in $T h\left(\Lambda^{o}\right)$, then it is strictly included in $T h\left(\Lambda^{o}\right)$. Namely, there exist two $\lambda$-terms $X$ and $Z$ such that

$$
T h\left(\Lambda^{o}\right) \vdash X=Z,
$$

while

$$
\operatorname{Th}(\mathbf{C}) \nvdash X=Z
$$

for every continuous model $\mathbf{C}$ such that $T h(\mathbf{C}) \subseteq T h\left(\Lambda^{o}\right)$. The complexity of the proof comes from the fact that (6) is established syntactically, while (7) is a consequence of the existence of non-sequential functions within the set of all Scott continuous functions.

Following a method similar to that of Honsell and Ronchi della Rocca [1992], Gouy [1995] proved the incompleteness of the stable semantics. Other more semantic proofs of incompleteness for the continuous, stable and hypercoherence semantics (i.e., a subclass of the strongly stable semantics introduced by Ehrhard [1993]) can be found in Bastonero [1998] and Bastonero and Gouy [1999] and are briefly described in the following. The Park model $\mathbf{P}$ was first defined in the framework of continuous semantics. It is a variant of the Scott model $D_{\infty}$, but with a very different equational theory. This model has a stable analogue $\mathbf{P}_{s}$ (which was defined by Honsell and Ronchi della Rocca [1990]), and a strongly stable analogue $\mathbf{P}_{f s}$ (defined by Bastonero and Gouy [1999]). It is possible to give a semantic proof of incompleteness for the continuous semantics by using the Park stable model $\mathbf{P}_{s}$. Bastonero and Gouy [1999] prove that 
the $\lambda$-terms $X$ and $Z$, considered by Honsell and Ronchi della Rocca [1992], are distinct in all continuous models $\mathbf{C}$ satisfying the following conditions:

(i) $\mathbf{C}$ is extensional,

(ii) $\Omega \Omega=\Omega$,

(iii) $\lambda x . \Omega(\Omega x)=\Omega$,

(iv) $\Omega \neq \lambda x . \Omega$.

The Park stable model $\mathbf{P}_{s}$ satisfies the above conditions (i)-(iv) and equalizes $X$ and $Z$ (see Bastonero and Gouy [1999, Thm. 4.1]). This proves that no continuous model has the theory of $\mathbf{P}_{s}$.

The incompleteness of the stable semantics was proven by Bastonero and Gouy in a similar way. They show that the same $\lambda$-terms $X$ and $Z$ are distinct in all stable models $\mathbf{C}$ satisfying the following conditions:

(a) $\mathbf{C}$ is extensional,

(b) $\Omega \Omega=\Omega$,

(c) $\Omega(\lambda x . \Omega) \neq \Omega$.

The Park strongly stable model $\mathbf{P}_{f s}$ satisfies the above three conditions (a)-(c) and equalizes $X$ and $Z$ (see Bastonero and Gouy [1999, Thm. 5.1]). This proves that no stable model has the theory of $\mathbf{P}_{f s}$.

The same technique was applied by Bastonero [1998] to prove the incompleteness of the hypercoherence semantics. He shows that there exist two $\lambda$-terms, $F$ and $G$, that are distinct in all hypercoherent models satisfying a suitable set $\mathcal{P}$ of constraints. Then, by using the technique of forcing, Bastonero builds a continuous model $\mathbf{C}$ that satisfies the constraints $\mathcal{P}$ and equalizes $F$ and $G$. This proves that no hypercoherent model has the theory of $\mathbf{C}$.

\section{CONCLUSION}

In this article, we have introduced a new technique to prove in a uniform way the incompleteness of all the denotational semantics of lambda calculus which have been proposed so far, including the strongly stable one, whose incompleteness had been conjectured by Bastonero and Gouy [1999] and by Berline [2000]. We apply this technique to prove the incompleteness of any semantics of lambda calculus given in terms of the following classes of models: (i) Partially ordered models with a bottom element; (ii) Partially ordered models with a finite number of connected components; (iii) Co-connected topological models. Our incompleteness theorems remove the belief that complete partial orders are intrinsic to models of the lambda calculus, and that the incompleteness of the continuous semantics is only due to the richness of the structure of the Scott continuous functions. Instead, the incompleteness is also due to the richness of the structure of the lambda theories.

\section{ACKNOWLEDGMENTS}

The author wishes to thank Gordon Plotkin and the referees for helpful comments and suggestions. 


\section{REFERENCES}

Abramsky, S. 1991. Domain theory in logical form. Ann. Pure Appl. Logic 51, 1-77.

Abramsky, S. ANd ONG, C. 1993. Full abstraction in the lazy $\lambda$-calculus. Inf. Comput. 105, 159-267.

Barendregt, H. 1984. The Lambda Calculus: Its Syntax and Semantics. Number 103 in Studies in Logic. North-Holland Publishing Co., Amsterdam, The Netherlands.

BAstonero, O. 1998. Equational incompleteness and incomparability results for $\lambda$-calculus semantics. Manuscript.

Bastonero, O. And Gouy, X. 1999. Strong stability and the incompleteness of stable models of $\lambda$-calculus. Ann. Pure Appl. Logic 100, 247-277.

Bentz, W. 1999. Topological implications in varieties. Algebra Univ. 42, 9-16.

BerLINe, C. 2000. From computation to foundations via functions and application: The $\lambda$-calculus and its webbed models. Theoret. Comput. Sci. 249, 81-161.

Berry, G. 1978. Stable models of typed lambda-calculi. In Proceedings of the 5th International Colloqium on Automata, Languages and Programming. Lecture Notes in Computer Science, vol. 62. Springer-Verlag, Berline, Germany.

Bucciarelli, A. ANd Ehrhard, T. 1991. Sequentiality and strong stability. In Proceedings of the 6th Annual IEEE Symposium on Logic in Computer Science. IEEE Computer Society Publications, Los Alamitos, Calif., 138-145.

Coleman, J. 1996. Separation in topological algebras. Algebra Univ. 35, 72-84.

Coleman, J. 1997. Topological equivalents to n-permutability. Algebra Univ. 38, 200-209.

CurRY, H. B. 1930. Grundlagen der kombinatorischen logik. Amer. J. Math. 52, 509-536.

Curry, H. B. And Feys, R. 1958. Combinatory Logic, Vol. I. North-Holland Publishing Co., Amsterdam, The Netherlands.

EHRHARD, T. 1993. Hypercoherences: A strongly stable model of linear logic. Math. Struct. Comput. Sci. 2, 365-385.

Gouy, X. 1995. Etude des théories équationnelles et des propriétés algébriques des modéles stables du $\lambda$-calcul. Ph.D. dissertation. Université de Paris 7, Paris, France.

Hofmann, K. AND Mislove, M. 1995. All compact Hausdorff lambda models are degenerate. Funda. Inf. $22,23-52$.

Honsell, F. and Ronchi della Rocca, S. 1990. Reasoning about interpretation in qualitative $\lambda$ models. In Programming Concepts and Methods, M. Broy and C. Jones, Eds. Elsevier Science Publishing Co., Amsterdam, The Netherlands, 505-521.

Honsell, F. AND RonCHI DELLA RocCA, S. 1992. An approximation theorem for topological $\lambda$-models and the topological incompleteness of $\lambda$-calculus. J. Comput. Syst. Sci. 45, 49-75.

Johnstone, P. 1982. Stone Spaces. Cambridge University Press, Cambridge, Mass.

KERTH, R. 1998. Isomorphism and equational equivalence of continuous lambda models. Stud. Logica 61, 403-415.

Kerth, R. 2001. On the construction of stable models of $\lambda$-calculus. Theoret. Comput. Sci. 269, $23-46$.

Lusin, S. And Salibra, A. 2003. A note on absolutely unorderable combinatory algebras. J. Logic Comput. to appear.

Meyer, A. 1982. What is a model of the lambda calculus? Inf. Cont. 52, 87-122.

Mislove, M. 1998. Topology, domain theory and theoretical computer science. Top. Appl. 89, 3-59.

Pigozzi, D. AND SALIBRA, A. 1998. Lambda abstraction algebras: coordinatizing models of lambda calculus. Fund. Inf. 33, 149-200.

Plotkin, G. 1993. Set-theoretical and other elementary models of the $\lambda$-calculus. Theoret. Comput. Sci. 121, 351-409.

Plotkin, G. 1996. On a question of H. Friedman. Inf. Comput. 126, 74-77.

SAlibra, A. 2000. On the algebraic models of lambda calculus. Theoret. Comput. Sci. 249, 197240.

SAlibra, A. 2001a. A continuum of theories of lambda calculus without semantics. In Proceedings of the 16th Annual IEEE Symposium on Logic in Computer Science (LICS'01). IEEE Computer Society Publications, Los Alamitos, Calif., 334-343.

SAlibra, A. 2001b. Towards lambda calculus order-incompleteness. In Workshop on Böhm theorem: Applications to Computer Science Theory (BOTH 2001). Electronics Notes in Theoretical 
Computer Science, vol. 50. Elsevier Science Publishing Company, Amsterdam, The Netherlands, 147-160.

Schönfinkel, M. 1924. Über die bausteine der mathematischen logik. Math Anal. 92, 305-316. Sсотт, D. 1972. Continuous lattices. In Toposes, Algebraic geometry and Logic, F. Lawvere, Ed. Lecture Notes in Computer Science, Vol. 274. Springer-Verlag, Berlin, Germany, 97-136.

Sсотт, D. 1980. Lambda calculus: Some models, some philosophy. In The Kleene Symposium, H. K. J. Barwise and K. Kunen, Eds. Number 101 in Studies in Logic. North-Holland Publishing Co., Amsterdam, The Netherlands, 97-136.

SELINGER, P. 1996. Order-incompleteness and finite lambda models. In Proceedings of the 11th Annual IEEE Symposium on Logic in Computer Science. IEEE Computer Society Publications, Los Alamitos, Calif.

SELINGER, P. 1997. Functionality, polymorphism, and concurrency: a mathematical investigation of programming paradigms. Ph.D. dissertation, University of Pennsylvania.

Steen, L. And Seebach, J. 1978. Counterexamples in topology. Springer-Verlag, Berlin, Germany. Świerczkowski, S. 1964. Topologies in free algebras. Proc. London Math. Soc. 3, 566-576.

TAYLOR, W. 1977. Varieties obeying homotopy laws. Canad. Journal Math. 29, 498-527.

URsinI, A. 1994. On subtractive varieties, I. Algebra Univ. 31, 204-222.

VIsser, A. 1980. Numerations, $\lambda$-calculus and arithmetic. In To H.B. Curry: Essays on Combinatory Logic, Lambda-Calculus and Formalism, J. Hindley and J. Seldin, Eds. Academic Press, New York, 259-284.

Received October 2001; revised August 2002; accepted October 2002 